\title{
Qunatum Halo States in Helium Tetramers
}

\author{
Petar Stipanović, ${ }^{*, \dagger}$ Leandra Vranješ Markić, ${ }^{\dagger}$ and Jordi Boronat ${ }^{\ddagger}$
}

Faculty of Science, University of Split, Ruđera Boškovića 33, HR-21000 Split, Croatia, and

Departament de Física, Campus Nord B4-B5, Universitat Politècnica de Catalunya,

E-08034 Barcelona, Spain

E-mail: pero@pmfst.hr

\footnotetext{
${ }^{*}$ To whom correspondence should be addressed

${ }^{\dagger}$ Faculty of Science, University of Split, Ruđera Boškovića 33, HR-21000 Split, Croatia

${ }^{\ddagger}$ Departament de Física, Campus Nord B4-B5, Universitat Politècnica de Catalunya, E-08034 Barcelona, Spain
} 


\begin{abstract}
Universality of quantum halo states enables comparison of systems from different fields of physics, as demonstrated in two- and three-body clusters. In the present work, we studied weakly-bound helium tetramers in order to test whether some of these four-body realistic systems qualify as halos. Their ground-state binding energies and structural properties were thoroughly estimated using the diffusion Monte Carlo method with pure estimators. Helium tetramer properties proved to be less sensitive on the potential model than previously evaluated trimer properties. We predict the existence of the realistic four-body halo ${ }^{4} \mathrm{He}_{2}{ }^{3} \mathrm{He}_{2}$, while ${ }^{4} \mathrm{He}_{4}$ and ${ }^{4} \mathrm{He}_{3}{ }^{3} \mathrm{He}$ are close to the border and thus can be used as prototypes of quasi-halo systems. Our results could be tested by experimental determination of tetramers' structural properties, using a similar setup to the one developed for the study of helium trimers.
\end{abstract}

\title{
Keywords
}

halo states, tetramers, helium clusters, quantum Monte Carlo

\section{Introduction}

Quantum mechanics predicts universal features of weakly bound and spatially extended fewbody systems. The Efimov effect ${ }^{1}$ in the quantum three-body problem is probably one of the most famous. An infinite series of three-body geometrically spaced and stable excited states was predicted to appear when a third particle is added to a pair of bosons that are on the edge of binding. Experimental evidence ${ }^{2}$ for Efimov quantum states in an ultracold trapped gas of cesium atoms caused a tremendous impact in the field. Furthermore, Efimov signatures were also encountered ${ }^{3,4}$ in four-body clusters.

Two- and three-body halos, whose wave functions extend significantly to classically for-

bidden regions of space, experience universal behavior. ${ }^{5-7}$ Such systems do not see details of 
short-range interactions and their properties can be predicted with any potential that has the same integral properties. Scale invariance noticed in nuclei ${ }^{5}$ proved to be applicable also to small atomic clusters. ${ }^{5,7}$

Helium atoms, which experience an extremely weak van der Waals interparticle interaction are a common ingredient of molecular quantum halos. ${ }^{7}$ Existence of the smallest clusters, dimer ${ }^{4} \mathrm{He}_{2}$, trimers ${ }^{4} \mathrm{He}_{3}$ and ${ }^{4} \mathrm{He}_{2}{ }^{3} \mathrm{He}$, and tetramers ${ }^{4} \mathrm{He}_{4},{ }^{4} \mathrm{He}_{3}{ }^{3} \mathrm{He}$ and ${ }^{4} \mathrm{He}_{2}{ }^{3} \mathrm{He}_{2}$, was predicted theoretically ${ }^{8-11}$ and confirmed experimentally. ${ }^{12-18}$ Their stability is affected by the different mass and quantum statistics of helium isotopes, ${ }^{4} \mathrm{He}$ and ${ }^{3} \mathrm{He}$. Both helium dimers and trimers played a significant role in the research ${ }^{7}$ of universal scaling laws of energy and size of two- and three-body halos.

For many years, ${ }^{19}$ the helium trimer ${ }^{4} \mathrm{He}_{3}$ has attracted the attention from both theory and experiment as one of the most promising candidates for observing Efimov states. ${ }^{1}$ An important aspect, from the theoretical side, has been the design of many sophisticated nonrelativistic Born-Oppenheimer $\left(V_{\mathrm{BO}}\right)$ He-He potentials, such as those given in Refs. 20 and 21. Recently, Przybytek et al. calculated ${ }^{22}$ and Cencek et al. analyzed ${ }^{23}$ the main post- $V_{\mathrm{BO}}$ physical effects, i.e., the adiabatic, relativistic, quantum electrodynamics, and retardation contributions. At the same time, experimental work managed to develop techniques able to get insight into the helium cluster structure. Recently, pair and angular distribution functions of helium trimers ${ }^{4} \mathrm{He}_{3}$ and ${ }^{4} \mathrm{He}_{2}{ }^{3} \mathrm{He}$ were measured ${ }^{12}$ by means of Coulomb explosion imaging. In this way, the ground state of ${ }^{4} \mathrm{He}_{2}{ }^{3} \mathrm{He}$ was experimentally confirmed ${ }^{12}$ to be halo. In 2015, a remarkable experiment confirmed the Efimov nature of the elusive excited state of the ${ }^{4} \mathrm{He}_{3}$ trimer. ${ }^{24}$ The measured structure data of the trimer ${ }^{12,24}$ were then used for an accurate evaluation ${ }^{25}$ of different He-He potentials.

Regarding four-body systems, linear correlations between the ${ }^{4}$ He tetramer and trimer binding energies were found for different potentials. ${ }^{26}$ In addition, theoretical work also studied universality ${ }^{27}$ in Efimov-associated ${ }^{4}$ He tetramers.

To our knowledge, a thorough analysis of the possibility that a tetramer can be qualified 
as a halo state has not been made yet. Approximate analysis ${ }^{29}$ of the tetramers ${ }^{4} \mathrm{He}_{2}(\mathrm{~T} \downarrow)_{2}$ and ${ }^{4} \mathrm{He}(\mathrm{T} \downarrow)_{3}$ modeled with an old and weakly attractive version of the He-T $\downarrow$ potential opened the possibility that those tetramers could be in a halo state. Four- and five-body systems with no bound subsystems, called Brunnian states, were investigated in Ref. 30 for some model interaction potentials. Their probability distributions were on average found in classically forbidden regions of space resulting in universal features. In addition, that analysis pointed that ground states for more than five particles forming a bound cluster were probably nonuniversal. However, no realistic molecular system was identified as Brunnian.

In the present work, we have chosen to study helium tetramers as candidates for halos because of their small mass, weak binding and experimental feasibility. They can not be classified as Brunnian states because they have at least one subsystem which is self-bound, ${ }^{4} \mathrm{He}_{2}$. Since ${ }^{4} \mathrm{He}^{3} \mathrm{He}_{3}$ is not stable, Jensen et al. suggested ${ }^{4} \mathrm{He}_{2}{ }^{3} \mathrm{He}_{2}$ as the most likely candidate to be halo, but stressed that the final decision would require further and accurate analysis. Furthermore, it was not tested how different theoretical potentials affect the helium tetramer structural properties, which are expected to be measured soon. ${ }^{24}$ Specifically, the structure of the most weakly bound helium tetramer ${ }^{4} \mathrm{He}_{2}{ }^{3} \mathrm{He}_{2}$ was not investigated.

In this work, we report how different potentials and their corrections affect the groundstate energy and structural properties of the helium tetramers. Furthermore, we test their halo nature. In the next Section, we introduce the diffusion Monte Carlo method (DMC) ${ }^{31}$ and discuss the trial wave functions used for importance sampling. After results are discussed and compared with other theoretical works, we comprise a summary of the work and an account of the main conclusions.

\section{Theoretical Method}

Both binding energy and structural properties of quantum clusters, which interact with a given potential, can be exactly estimated up to statistical errors using the DMC method 
with pure estimators. ${ }^{32}$ We first present the selected interaction potential models and then discuss the basic features of the method.

\section{Interaction potential models}

We modeled the interaction of $\mathrm{He}$ atoms in all stable helium tetramers ${ }^{4} \mathrm{He}_{4},{ }^{4} \mathrm{He}_{3}{ }^{3} \mathrm{He}$, and ${ }^{4} \mathrm{He}_{2}{ }^{3} \mathrm{He}_{2}$ using pair potential models that were obtained using different methods and approximation levels. Among the most frequently used potential models, we selected a few.

First, we took the semi-empirical HFDB ${ }^{20}$ model where the B-type of Hartree-Fock model with damped dispersion (HFD) was adapted by Aziz et al. to a selected set of experimental and theoretical results.

The most sophisticated theoretical model PCKLJS (the authors ${ }^{22}$ acronym) was also considered. Jeziorska et al. ${ }^{33}$ combined supermolecular data and the symmetry-adapted perturbation theory in order to obtain a fitted analytic function for the He-He potential. Afterwards, Przybytek et al. ${ }^{22}$ included leading order coupling of the electronic and nuclear motion, i.e., adiabatic corrections, relativistic and quantum electrodynamics contributions. Also, Casimir-Polder ${ }^{34}$ retardation effects (Ret) were included in PCKLJS. The resulting PCKLJS potential is similar to the HFDB because some corrections cancel each other.

A third model used in our work is the one derived from perturbation theory by Tang, Toennies and Yiu (TTY), ${ }^{21}$ and TTY+Ret model, i.e. TTY without and with retardation. Among several options, the latter was surprisingly the best one in reproducing the experimentally determined helium trimer structural properties. ${ }^{25}$

The selected potentials are compared in Fig. 1. The most visible differences between them are in the vicinity of the minimum, which is zoomed in the inset.

It was shown ${ }^{25,35}$ that three-body interactions have negligible effect on the properties of helium trimers. Thus, we did not take them into account in the present work. Based on a recent comparison of theoretical predictions with trimer experimental measurements ${ }^{25}$ all other frequently used He-He potential models are expected to give similar estimates of 


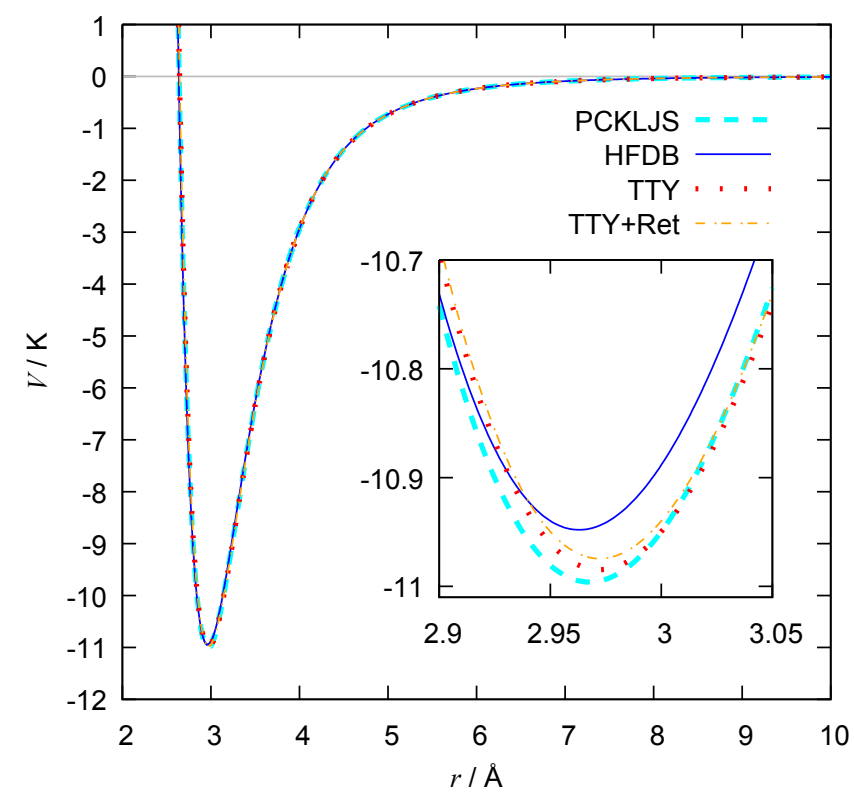

Figure 1: Attractive part of He-He potentials PCKLJS, ${ }^{22} \mathrm{HFDB},{ }^{20} \mathrm{TTY}^{21}$ and TTY + Ret $^{22}$ as a function of separation $r$ between He atoms.

tetramer characteristics.

\section{Diffusion Monte Carlo method}

Helium clusters were studied at zero absolute temperature using the DMC method. The DMC method solves stochastically the Schrödinger equation written in imaginary time $\tau=$ $i t / \hbar$

$$
-\frac{\partial \Psi(\mathbf{R}, \tau)}{\partial \tau}=\left(H-E_{\mathrm{r}}\right) \Psi(\mathbf{R}, \tau)
$$

applying reasonable approximations for the Green's function when imaginary time step $\Delta \tau \rightarrow$. In Eq. (1), $E_{\mathrm{r}}$ is a reference energy and the walker $\boldsymbol{R} \equiv\left(\boldsymbol{r}_{1}, \boldsymbol{r}_{2}, \boldsymbol{r}_{3}, \boldsymbol{r}_{4}\right)$ collectively denotes the positions of the tetramer's constituents. The Hamiltonian

$$
H=-\sum_{i=1}^{4} \frac{\hbar^{2}}{2 m_{i}} \nabla_{i}^{2}+\sum_{\substack{i, j=1 \\ i<j}}^{4} V\left(r_{i j}\right)
$$


is given as a sum of the kinetic energy operators of all the particles and all the He-He pair interactions $V$. Constants $\hbar^{2} m_{i}^{-1}$ were calculated using the best available data from the NIST database, for ${ }^{4} \mathrm{He}$ and ${ }^{3} \mathrm{He}$ equal to $12119.28157 \mathrm{mK} \AA^{2}$ and $16083.62212 \mathrm{mK} \AA^{2}$, respectively.

In order to reduce the variance of the calculation to a manageable level, we applied the same approach as in the study of helium trimer properties, ${ }^{25}$ i.e., the Schrödinger equation (1) was rewritten for the mixed distribution $\Phi(\boldsymbol{R}, \tau)=\Psi(\boldsymbol{R}, \tau) \psi(\boldsymbol{R})$ which is represented by a set of walkers $\{\boldsymbol{R}\}$. The trial wave function, which guides the diffusion process, was optimized by the variational Monte Carlo (VMC) method, minimizing the energy and its variance. For the trial wave function, a Jastrow type was used,

$$
\psi(\boldsymbol{R})=\prod_{i<j}^{4} F_{k}\left(r_{i j}\right)
$$

i.e., a product of two-body correlation functions

$$
F_{k}(r)=\frac{1}{r} \exp \left[-\left(\frac{\alpha_{k}}{r}\right)^{\gamma_{k}}-s_{k} r\right]
$$

where $r$ is the interparticle distance and $k=44,43$ denotes ${ }^{4} \mathrm{He}-{ }^{4} \mathrm{He}$ and ${ }^{4} \mathrm{He}^{-}{ }^{3} \mathrm{He}$ pairs, respectively. Variational parameters $\alpha_{k}$ and $\gamma_{k}$ describe short-range correlations, while $s_{k}$ is used for the long-range ones. The optimization of the trial wave functions was done for all clusters using the HFDB interaction potential. Due to the similarity between the considered potential models, the same parameters were used for a particular cluster in all further DMC simulations.

In the case of the pure ${ }^{4} \mathrm{He}$ tetramer, parameters $\alpha_{44}=2.82 \AA, \gamma_{44}=4.14$ and $s_{44}=$ $0.02 \AA^{-1}$ lowered the VMC energy to $90 \%$ of the DMC energy. Swapping one ${ }^{4} \mathrm{He}$ atom by ${ }^{3} \mathrm{He}$ the optimal parameters for the ${ }^{4} \mathrm{He}-{ }^{4} \mathrm{He}$ correlations did not change, while swapping two of them changed the parameters to $\alpha_{44}=2.83 \AA$ and $s_{44}=0.01 \AA^{-1}$. Optimal parameters for the ${ }^{4} \mathrm{He}-{ }^{3} \mathrm{He}$ correlations were $\alpha_{43}=2.87,2.90 \AA, \gamma_{43}=3.74,3.6$, and $s_{43}=0.0006 \AA^{-1}$ 
for ${ }^{4} \mathrm{He}_{3}{ }^{3} \mathrm{He}$ and ${ }^{4} \mathrm{He}_{2}{ }^{3} \mathrm{He}_{2}$, respectively. The ${ }^{3} \mathrm{He}^{-}{ }^{3} \mathrm{He}$ correlations in ${ }^{4} \mathrm{He}_{2}{ }^{3} \mathrm{He}_{2}$ were similar to the ${ }^{4} \mathrm{He}^{-}{ }^{3} \mathrm{He}$ correlations. The difference between VMC and DMC results increased when ${ }^{4} \mathrm{He}$ atoms were swapped by ${ }^{3} \mathrm{He}$ ones; for substitutions of one and two atoms VMC reached approximately $80 \%$ and $30 \%$ of the DMC energy, respectively.

We used a DMC method ${ }^{31}$ which is accurate to second order in the time step $\Delta \tau$, $E_{\mathrm{DMC}}(\Delta \tau)=E+k_{E}(\Delta \tau)^{2}$. Both the time step dependence and the mean walker population were studied carefully in order to eliminate any residual bias. For all studied tetramers 5000 walkers proved to be enough. The DMC energies $E_{\mathrm{DMC}}(\Delta \tau)$ were calculated for different time steps (from $6 \times 10^{-4} \mathrm{~K}^{-1}$ to $18 \times 10^{-4} \mathrm{~K}^{-1}$ ) and the final results were derived by extrapolation to zero time step.

The expectation value of an operator which does not commute with the Hamiltonian $H$ can be accurately calculated using pure estimators. ${ }^{32}$ For the mean square root of pair distances $r_{i j}$, density profiles $\rho(r)$ and the pair distributions functions $P(r)$ and $P_{2}\left(r_{i j}, r_{k l}\right)$ we verified that the chosen block size is large enough to guarantee asymptotic offspring, i.e., to correct the bias coming from the choice of the trial wave function. All presented results were obtained using $10^{5}$ steps per block, although some properties converged even for 4 times smaller block sizes.

\section{Results and Discussion}

\section{Binding energies}

Some helium tetramer properties were studied in the past using different methods and interatomic potentials. In agreement with the literature, we predict stability of three isotopic combinations, which include no more than two fermions: ${ }^{4} \mathrm{He}_{4},{ }^{4} \mathrm{He}_{3}{ }^{3} \mathrm{He}$ and ${ }^{4} \mathrm{He}_{2}{ }^{3} \mathrm{He}_{2}$. Tetramers with more than two ${ }^{3} \mathrm{He}$ atoms, which require the inclusion of Fermi statistics, are not studied here because it is not expected that they form a self-bound state. ${ }^{9,13}$ Helium tetramer ground-state energies $E$ calculated using different potential models $V$ are reported 
in Table 1. DMC statistical errors $\sigma_{E}$ are given in parenthesis. Results from our work are given in rows starting with the acronym of the potential model $V$ used in the calculation. In addition, we report for comparison the results from the literature that were obtained with potentials given in the preceding row.

Table 1: Ground state energies $E$ of He tetramers obtained using different He-He potentials $V$. Below appropriate row comparison is made with values from given references.

\begin{tabular}{lccc}
\hline & \multicolumn{3}{c}{$-E / \mathrm{mK}$} \\
\cline { 2 - 4 }$V /$ Ref. & ${ }^{4} \mathrm{He}_{4}$ & ${ }^{4} \mathrm{He}_{3}{ }^{3} \mathrm{He}$ & ${ }^{4} \mathrm{He}_{2}{ }^{3} \mathrm{He}_{2}$ \\
\hline TTY+Ret & $552.5(7)$ & $291.7(5)$ & $98.0(7)$ \\
TTY & $559.3(5)$ & $297.1(5)$ & $101.4(8)$ \\
Ref. 8 & $558.4(4)$ & $296.7(3)$ & $99.7(9)$ \\
PCKLJS & $573.9(6)$ & $307.8(5)$ & $108.7(8)$ \\
Ref. 26 & $573.9(6)$ & & \\
HFDB & $577.6(3)$ & $310.8(6)$ & $110.3(9)$ \\
Ref. 9 & $577(6)$ & $303(4)$ & $110(5)$ \\
\hline
\end{tabular}

Our TTY and HFDB results are equal to the DMC values from Ref. 8 and Ref. 9 up to one standard deviation; only the ${ }^{4} \mathrm{He}_{3}{ }^{3} \mathrm{He} \mathrm{HFDB}$ values are equal within two errorbars. Hiyama and Kamimura calculated ${ }^{26}$ the binding energy for the pure He tetramer, ${ }^{4} \mathrm{He}_{4}$, using the Gaussian expansion method for ab initio variational calculations of few-body systems and seven different potentials. The reported values ${ }^{26,27}$ for TTY, PCKLJS and HFDB are equal to our estimates obtained by the DMC method. Their weakest potential model LM2M2 ${ }^{36}$ predicted $-559.22 \mathrm{mK}^{26}\left(-558.98 \mathrm{mK}^{38}\right.$ for $\left.\hbar^{2} / m=12.12 \mathrm{~K} \AA^{2}\right)$ that is equal to our TTY results, but a bit stronger than our TTY + Ret prediction, as expected. Moreover, they estimated $^{26}$ the effects of different corrections included in the full PCKLJS He-He potential. Adiabatic correction strengthens the binding, which is weakened after adding appropriate retardation correction, further weakened after including relativistic corrections and finally strengthened applying level appropriate retardation. Further, it is increased including quantum electrodynamics effects, but decreased applying level appropriate retardation. Thus, our HFDB energy is between values of the full PCKLJS (Tab. 1) and the reported value for the Born-Oppenheimer part of PCKLJS without corrections, $-578.10 \mathrm{mK} .{ }^{26} \mathrm{Our}^{4} \mathrm{He}_{2}{ }^{3} \mathrm{He}_{2}$ 
HFDB and TTY ground state energies also confirm previous ${ }^{39}$ DMC estimates.

There are no results in literature for the TTY + Ret model which includes retardation effects in TTY. Also, mixed helium tetramers were not estimated using the currently most accurate He-He potential, PCKLJS.

If one or two ${ }^{4} \mathrm{He}$ atoms in ${ }^{4} \mathrm{He}_{4}$ are swapped by the lighter isotope ${ }^{3} \mathrm{He}$, the binding energy is reduced by $46-47 \%$ or $81-82 \%$, respectively. Higher differences correspond to the weaker binding. Among the potentials considered here, HFDB predicts the strongest binding, being stronger by $0.8-1.6 \%$ than PCKLJS. TTY and TTY + Ret predict weaker binding than PCKLJS, respectively by $2-7 \%$ and $4-10 \%$.

\section{Structural properties}

Challenged by the recent experimentally measured ${ }^{12,24,37}$ distributions of helium trimers ${ }^{4} \mathrm{He}_{3}$ and ${ }^{4} \mathrm{He}_{2}{ }^{3} \mathrm{He}$, we determined the structure of the ground state for all helium tetramers. In order to extract exact ground state distribution functions from the DMC sampled positions we used pure estimators. Results for the distribution functions are shown in figures 2 , and 3 . Functions obtained using various potential models are distinguished by different line types and widths.

In Fig. 2, we compared two-body distributions functions $P(r)$ estimated using PCKLJS,

HFDB, TTY and TTY + Ret potentials. Results for ${ }^{4} \mathrm{He}_{4},{ }^{4} \mathrm{He}_{3}{ }^{3} \mathrm{He}$ and ${ }^{4} \mathrm{He}_{2}{ }^{3} \mathrm{He}_{2}$ are shown in panels (a), (b) and (c) panels, respectively. When distribution functions of different pairs are presented in the same plot, top peaks of different line types stand for ${ }^{4} \mathrm{He}^{4}{ }^{4} \mathrm{He}$ separations. Although differences in the distribution functions for different potentials are barely visible for ${ }^{4} \mathrm{He}-{ }^{4} \mathrm{He}$, they are the more discernible the weaker binding is. In all shown cases only small differences are noticeable around the correlation peak. The weaker binding produces a lower correlation peak, which is shifted away from the potential minimum, and a longer tail at large distances. The effect is much more noticeable when comparing different clusters than comparing estimates obtained using different potential models. 


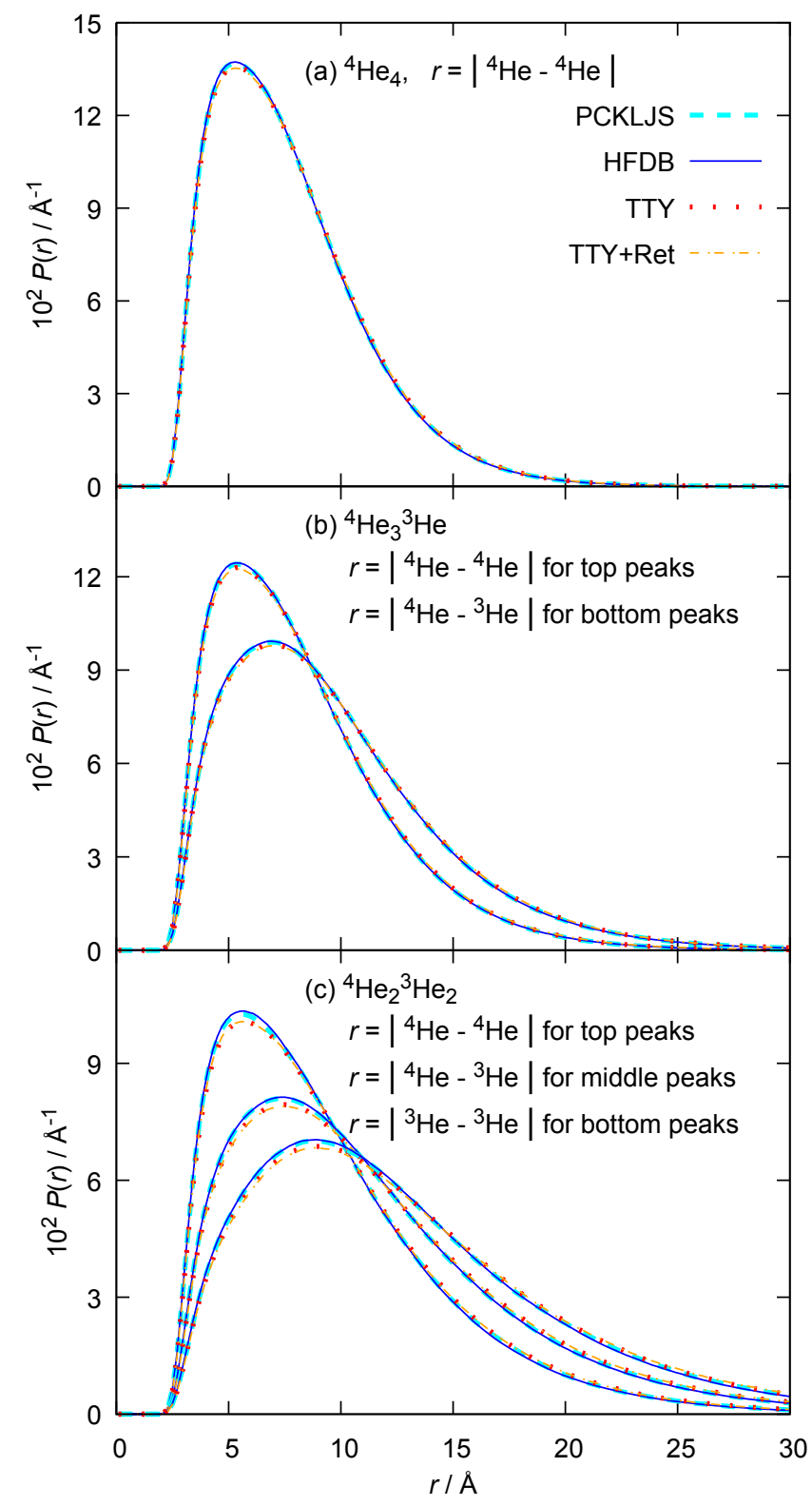

Figure 2: Distributions $P(r)$ of all He-He separations $r$ evaluated for different potentials in tetramers: (a) ${ }^{4} \mathrm{He}_{4}$; (b) ${ }^{4} \mathrm{He}_{3}{ }^{3} \mathrm{He}$; $(\mathrm{c}){ }^{4} \mathrm{He}_{2}{ }^{3} \mathrm{He}_{2}$. All $P(r)$ are normalized to $\int P(r) \mathrm{d} r=1$. 


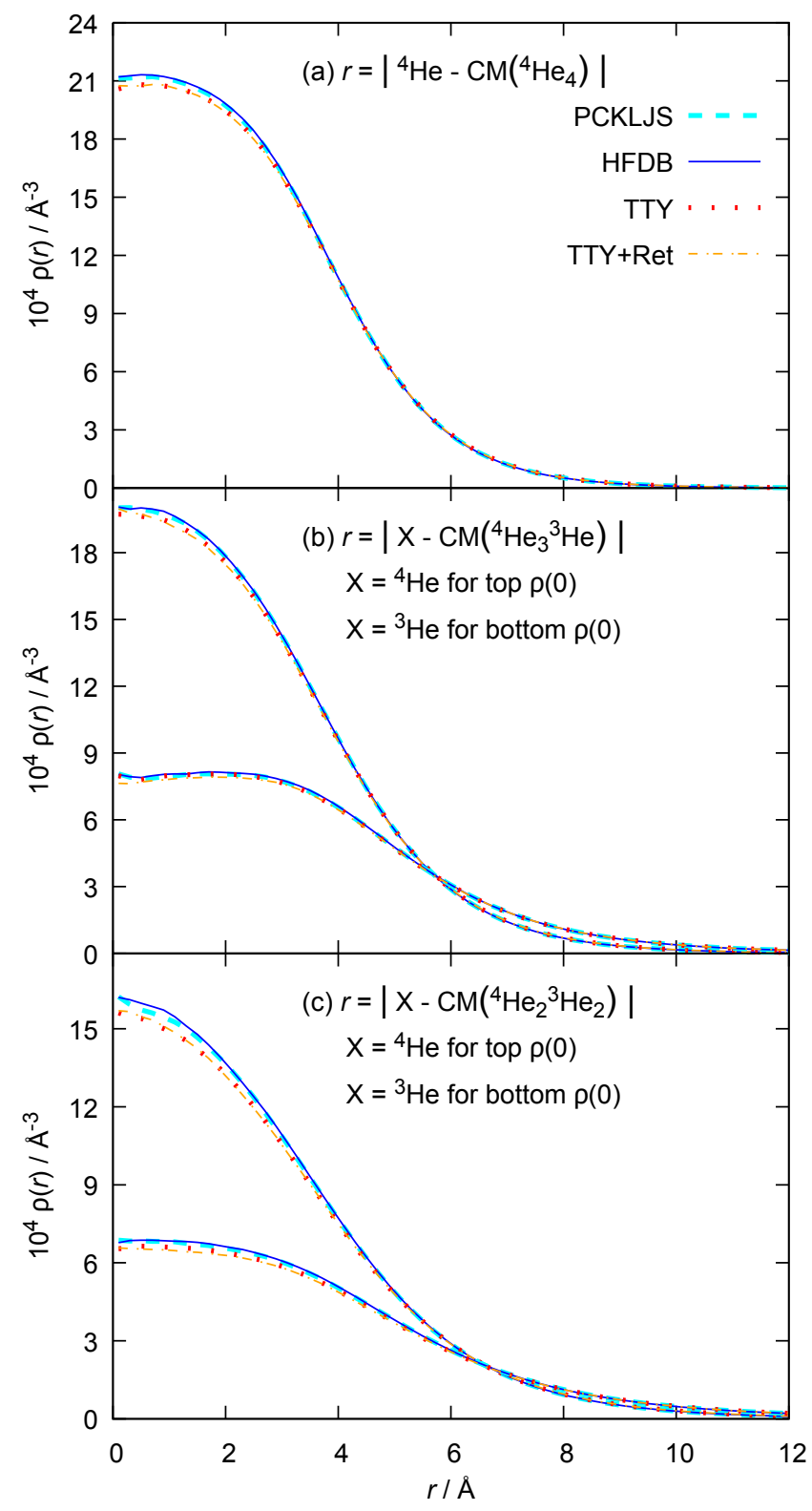

Figure 3: Density profiles $\rho(r)$ for ${ }^{4} \mathrm{He}$ and ${ }^{3} \mathrm{He}$, with respect to the center of mass $(\mathrm{CM})$ of $\mathrm{He}$ tetramers: (a) ${ }^{4} \mathrm{He}_{4}$; (b) ${ }^{4} \mathrm{He}_{3}{ }^{3} \mathrm{He}$; (c) ${ }^{4} \mathrm{He}_{2}{ }^{3} \mathrm{He}_{2}$. All $\rho(r)$ are normalized to $4 \pi \int \rho(r) r^{2} \mathrm{~d} r=1$. 
Similar to the previous, but slightly more pronounced behavior, is visible from the remaining groups of data in Fig. 2. Line groups in (b) with bottom peaks and (c) with middle peaks show distributions of ${ }^{4} \mathrm{He}-{ }^{3} \mathrm{He}$ separations in ${ }^{4} \mathrm{He}_{3}{ }^{3} \mathrm{He}$ and ${ }^{4} \mathrm{He}_{2}{ }^{3} \mathrm{He}_{2}$, while (c) with bottom peaks show ${ }^{3} \mathrm{He}-{ }^{3} \mathrm{He}$ distributions in ${ }^{4} \mathrm{He}_{2}{ }^{3} \mathrm{He}_{2}$.

All studied inter-atomic separations shown in Fig. 2 are very wide in comparison to the range of attractive visible part of the He-He potentials shown in Fig. 1. Regardless of the potential model, heavier pair is on average closer than a lighter pair. A vanishing short range part of $P(r)$ is consequence of the extremely repulsive short-range part of the He-He potentials.

In Fig. 3, density profiles $\rho(r)$ of He, with $r$ the distance to the center of mass, are shown for different interaction potentials. Different subfigures present different He tetramers. Group of lines in a subfigure which has higher, or only one $\rho(0)$, corresponds to ${ }^{4} \mathrm{He}$, while the remaining groups correspond to ${ }^{3} \mathrm{He}$. One can notice similar behavior as observed in the corresponding $P(r)$. The inner density of ${ }^{4} \mathrm{He}$ decreases as the number of ${ }^{3} \mathrm{He}$ atoms increases. The central part of the density profile is flatter for the larger number of ${ }^{4} \mathrm{He}$ present in the tetramer. The lighter isotope has a tendency to move to the surface but this effect is not so obvious due to small number of atoms. Shifting of the $\rho\left({ }^{3} \mathrm{He}\right)$ peak away from the center is barely noticeable in the case of the ${ }^{4} \mathrm{He}_{3}{ }^{3} \mathrm{He}$ tetramer. More apparent tendency of the ${ }^{3} \mathrm{He}$ atom to form Andreev states can be observed ${ }^{28}$ in structures of clusters that have much larger number of ${ }^{3} \mathrm{He}$ atoms. Qualitatively, the same behavior was noticed ${ }^{29}$ in clusters ${ }^{4} \mathrm{He}_{N}(\mathrm{~T} \downarrow)_{M}$ where $\mathrm{T} \downarrow$ appears instead of ${ }^{3} \mathrm{He}$.

$P(r)$ and $\rho(r)$ distributions in ${ }^{4} \mathrm{He}_{3}{ }^{3} \mathrm{He}$ modeled by TTY were already extracted ${ }^{28}$ from DMC sampled positions but using the approximated extrapolated estimator. Qualitatively, their results resemble the behavior that we observed in our estimates. Recently, $P(r)$ distributions were calculated ${ }^{38,40}$ using the newest He-He potential model PCKLJS, but only for ${ }^{4} \mathrm{He}_{4}$.

Diffuseness of helium tetramers is clearly visible from distributions $P_{2}\left(r_{i j}, r_{k l}\right)$ of ${ }^{i} \mathrm{He}-{ }^{j} \mathrm{He}$ 
distances in mixed helium tetramers ${ }^{4} \mathrm{He}_{2}{ }^{3} \mathrm{He}_{2}$ and ${ }^{4} \mathrm{He}_{3}{ }^{3} \mathrm{He}$. Results obtained using the most accurate He-He potential PCKLJS are shown in Fig. 4 where $x$-axis labels are given below bottom and above top row. Manifolded system's configuration arrangements are noticeable, i.e., for a particular $r_{i j}$ value a wide range of distances $r_{k l}$ has high probability of occurrence. Distributions are squeezed in direction of the axis belonging to the heavier pair. Diagonal extension in subfigure (b) indicates similar stretch of pairs $r_{43}$ and $r_{33}$. The bottom row compares $P_{2}\left(r_{44}, r_{43}\right)$ for two different tetramers. Among them, heavier has more compact distribution, but still extremely different from structural properties of classical systems ${ }^{41}$ $\mathrm{Ar}_{4}$ and $\mathrm{Ne}_{4}$.

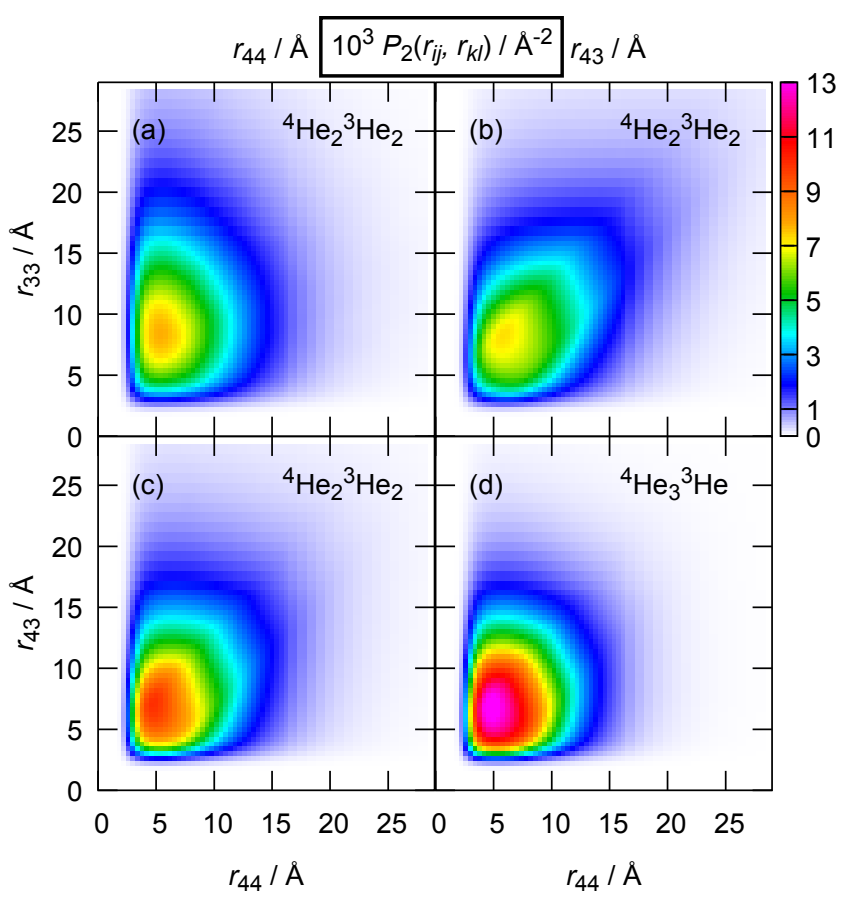

Figure 4: Distributions $P_{2}\left(r_{i j}, r_{k l}\right)$ of all ${ }^{i} \mathrm{He}-{ }^{j} \mathrm{He}$ separations $r_{i j}$ evaluated for PCKLJS potential in tetramers: (a)-(c) ${ }^{4} \mathrm{He}_{2}{ }^{3} \mathrm{He}_{2}$; (d) ${ }^{4} \mathrm{He}_{3}{ }^{3} \mathrm{He}$. All $P_{2}$ are normalized to $\iint P_{2}\left(r_{i j}, r_{k l}\right) \mathrm{d} r_{i j} \mathrm{~d} r_{k l}=1$.

\section{Quantum halo characteristics}

A quantum halo state is defined by the condition of being an extremely non-classical system, with high probability of being in a classically forbidden region. In a previous work, ${ }^{7}$ we 
established the convenient length scale for energy-size scaling for few-body halo systems having $N<4$ particles. The size of a system was measured ${ }^{5,7}$ by the mean square hyperradius

$$
\rho^{2}=\frac{1}{M m} \sum_{i<k}^{N} m_{i} m_{k}\left\langle r_{i k}^{2}\right\rangle
$$

where $m$ is an arbitrary mass unit, $m_{i}$ the mass of particle $i, M$ the total mass of the system, and $\left\langle r_{i k}^{2}\right\rangle$ is the mean square distance between particles $i$ and $k$. Further, the system size was scaled, $Y_{\rho}=\rho^{2} \rho_{0}^{-2}$, where $\rho_{0}^{2}$ is obtained using the squared characteristic length $R_{i k}^{2}$ instead of $\left\langle r_{i k}^{2}\right\rangle$ in Eq. (5). For $R_{i k}$ we took the width of a square well potential which is equivalent ${ }^{7}$ to the realistic potential $V\left(r_{i k}\right)$.

In the present work we extended the same definition to $N=4$ particles. Using the standard criterion, a halo state is the one with a scaled size $Y_{\rho}$ greater than $2 .{ }^{5}$ Values of $\left\langle r_{i k}^{2}\right\rangle$ were extracted by the pure estimators from the DMC-sampled positions. The value reported in Ref. 26 for ${ }^{4} \mathrm{He}_{4}$, modeled by PCKLJS, is equal to our estimate $69.7(5) \AA^{2}$. There are no more data in literature for other helium tetramers to compare. Regardless of the potential model, the mixed tetramer ${ }^{4} \mathrm{He}_{2}{ }^{3} \mathrm{He}_{2}$ has clear characteristics of four-body halo. Scaled size $Y_{\rho}$ ranges from 2.44 to 2.51 , where the higher values correspond to the less attractive potential (see Table 1). Although not above the limit, $Y_{\rho}$ values of ${ }^{4} \mathrm{He}_{3}{ }^{3} \mathrm{He}$ and ${ }^{4} \mathrm{He}_{4}, 1.57-1.61$ and $1.22-1.25$, respectively, indicate that a significant part of their wavefunction is in the classically forbidden region of space. Thus, it is expected that they will share some halo characteristics, as it was shown ${ }^{7}$ previously for trimers which are close to the halo edge. In this line, it is worth noticing the similarity between the structure of clusters ${ }^{4} \mathrm{He}_{2}{ }^{3} \mathrm{He}_{2}$ and ${ }^{4} \mathrm{He}_{3}{ }^{3} \mathrm{He}$ (bottom row of Fig. 4).

\section{Conclusions}

Using accurate diffusion Monte Carlo simulations we obtained the ground state energy and structural properties of all stable helium tetramers ${ }^{4} \mathrm{He}_{2}{ }^{3} \mathrm{He}_{2},{ }^{4} \mathrm{He}_{3}{ }^{3} \mathrm{He},{ }^{4} \mathrm{He}_{4}$. Structural 
properties were calculated by pure estimators in order to completely eliminate the bias of the trial wavefunction. Sensitiveness of estimated values was tested using four different potential models, including the frequently used models HFDB, TTY and the currently most accurate He-He potential PCKLJS. The TTY+Ret potential was used for the first time because it surprisingly proved ${ }^{25}$ to be in the best agreement with measurements of helium trimer properties. ${ }^{12,24}$

Our predictions for the ground-state energy are in excellent agreement with the most recent estimates. All structural properties of ${ }^{4} \mathrm{He}_{2}{ }^{3} \mathrm{He}_{2}$ were calculated for the first time. Noticeably, we observed four-body halo in ${ }^{4} \mathrm{He}_{2}{ }^{3} \mathrm{He}_{2}$ with large spread between all pairs of particles. This means it can not be approximated as an effective two- or three-body system, in which three or two particles would be closer together, effectively forming a single body, while the remaining particles would be further apart. It is worth noticing that the detection and characterization of such a system in nuclear physics is much more challenging due to unstable nature of the most favored systems consisting of a core and three neutrons. ${ }^{42}$ Other helium tetramers were found close to the halo edge and can approximately be considered as quasi-halos due to their wideness.

Sensitiveness of structural properties on the potential model was tested for the first time for all He tetramers. Unlike the case of helium trimers ${ }^{25}$ structural properties of helium tetramers showed to be much less sensitive to the potential model. Thus, if the expected future measurements could be accomplished without any biases, such as the temperature effects, we would expect very good agreement with our theoretical estimates.

Structure of Coulomb exploded argon and neon dimers, trimers, and tetramers was recently measured and the development of experimental techniques was announced for application on helium clusters. ${ }^{41}$ Afterwards, when helium trimer structural properties were measured, the approach was qualified as feasible for the four-body sector. ${ }^{24}$ Based on our predictions and the announcements from experimental groups we thus expect that the first molecular four-body quantum halo state could be experimentally confirmed in the near fu- 
ture.

\section{Acknowledgement}

This work has been supported in part by the Croatian Science Foundation under the project

number IP-2014-09-2452. J. B. acknowledge additional support by the MICINN-Spain, Grant No. FIS2014-56257-C2-1-P. The computational resources of the Isabella cluster at Zagreb University Computing Center (Srce), the HYBRID cluster at the University of Split, Faculty of Science and Croatian National Grid Infrastructure (CRO NGI) were used.

\section{References}

(1) Efimov, V. Energy levels arising from resonant two-body forces in a three-body system. Phys. Lett. 1970, 33B, 563-564.

(2) Kraemer, T.; Mark, M.; Waldburger, P.; Danzl, J. G.; Chin, C.; Engeser, B.; Lange, A. D.; Pilch, K.; Jaakkola, A.; Nägerl, H.-C. et al. Evidence for Efimov quantum states in an ultracold gas of caesium atoms. Nature 2006 440, 315-318.

(3) Hammer, H. W.; Platter, L. Universal properties of the four-body system with large scattering length. Eur. Phys. J. A 2007, 32, 113-120.

(4) von Stecher, J.; D'Incao, J. P.; Greene, Chris H. Signatures of universal four-body phenomena and their relation to the Efimov effect. Nature 2009, 5, 417-421.

(5) Jensen, A. S.; Riisager, K.; Fedorov, D. V.; Garrido, E. Structure and reactions of quantum halos. Rev. Mod. Phys. 2004, 76, 215-261.

(6) Riisager, K. Halos and related structures. Phys. Scr. 2013, T152, 014001-13.

(7) Stipanović, P.; Vranješ Markić, L.; Bešlić, I.; Boronat, J. Universality in Molecular Halo Clusters. Phys. Rev. Lett. 2014, 113, 253401-5. 
(8) Bressanini, D.; Morosi, G. Stability of ${ }^{3} \mathrm{He}_{2}{ }^{4} \mathrm{He}_{M}$ and ${ }^{3} \mathrm{He}_{3}{ }^{4} \mathrm{He}_{M} L=0,1$ Clusters. Few-Body Systems 2004, 34, 131-135.

(9) Guardiola, R.; Navarro, J. Stability chart of small mixed ${ }^{4} \mathrm{He}-{ }^{3} \mathrm{He}$ clusters. Phys. Rev. A 2003, 68, 055201-4.

(10) Whaley, K. B. Structure and reactions of quantum clusters. Int. Rev. Phys. Chem. 1994, 13, 41-84.

(11) Sola, E.; Casulleras, J.; Boronat, J. Ground-state energy and stability limit of ${ }^{3} \mathrm{He}$ droplets. Phys. Rev. B 2006, 73, 092515-4.

(12) Voigtsberger, J.; Zeller, S.; Becht, J.; Neumann, N.; Sturm, F.; Kim, H-K.; Waitz, M.; Trinter, F.; Kunitski, M.; Kalinin, A. et al. Imaging the structure of the trimer systems ${ }^{4} \mathrm{He}_{3}$ and ${ }^{3} \mathrm{He}^{4} \mathrm{He}_{2}$. Nat. Comm. 2014, 5, 5765-6.

(13) Kalinin, A.; Kornilov, O.; Schöllkopf, W.; Toennies, J. P. Observation of Mixed Fermionic-Bosonic Helium Clusters by Transmission Grating Diffraction. Phys. Rev. Lett. 2005, 95, 113402-4.

(14) Luo, F; McBane, G.; Kim, G.; Giese, C. F. The weakest bond: Experimental observation of helium dimer. J. Chem. Phys. 1993, 98, 3564-3567.

(15) Luo, F.; Giese, C. F.; Gentry, W. R. Direct measurement of the size of the helium dimer. J. Chem. Phys. 1996, 104, 1151-1154.

(16) Schöllkopf, W.; Toennies, J. P. Nondestructive mass selection of small van der Waals clusters, Science 1994, 266 1345-1348.

(17) Schöllkopf, W.; Toennies, J. P. The nondestructive detection of the helium dimer and trimer. J. Chem. Phys. 1996, 104, 1155-1158. 
(18) Grisenti, R. E.; Schöllkopf, W.; Toennies, J. P.; Hegerfeldt, G. C.; Köhler, T.; Stoll, M. Determination of the Bond Length and Binding Energy of the Helium Dimer by Diffraction from a Transmission Grating. Phys. Rev. Lett. 2000, 85, 2284-2287.

(19) Lim, T. K.; Duffy, S. K.; Damer, W. C. Efimov State in the ${ }^{4}$ He Trimer. Phys. Rev. Lett. 1977 38, 341-343.

(20) Aziz, R. A.; McCourt, F. R. W.; Wong, C. C. K. A new determination of the ground state interatomic potential for $\mathrm{He}_{2}$. Mol. Phys. 1987, 61, 1487-1511.

(21) Tang, K. T.; Toennies, J. P.; Yiu, C. L. Accurate Analytical He-He van der Waals Potential Based on Perturbation Theory. Phys. Rev. Lett. 1995, 74, 1546-1549.

(22) Przybytek, M.; Cencek, W.; Komasa, J.; Åtach, G.; Jeziorski, B.; Szalewicz, K. Relativistic and Quantum Electrodynamics Effects in the Helium Pair Potential. Phys. Rev. Lett. 2010, 104, 183003-4. See also supplementary material at http://link. aps .org/ supplemental/10.1103/PhysRevLett.104.183003, and the errata to this supplement in Phys. Rev. Lett. 2012, 108 129902-1.

(23) Cencek, W.; Przybytek, M.; Komasa, J.; Mehl, J. B.; Jeziorski, B.; Szalewicz, K. Effects of adiabatic, relativistic, and quantum electrodynamics interactions on the pair potential and thermophysical properties of helium. J. Chem. Phys. 2012, 136 22430331.

(24) Kunitski, M.; Zeller, S.; Voigtsberger, J.; Kalinin, A.; Schmidt, L. Ph. H.; Schöffler, M.; Czasch, A.; Schöllkopf, W.; Grisenti, R. E.; Jahnke, T.; Blume, D.; Dörner, R. Observation of the Efimov state of the helium trimer, Science 2015, 348, 551-555.

(25) Stipanović, P.; Vranješ Markić, L.; Boronat, J. Elusive structure of helium trimers. $J$. Phys. B: At. Mol. Opt. Phys. 2016, 49, 185101-11. 
(26) Hiyama, E.; Kamimura, M. Linear correlations between ${ }^{4}$ He trimer and tetramer energies calculated with various realistic ${ }^{4} \mathrm{He}$ potentials. Phys. Rev. A 2012, 85, 062505-10.

(27) Hiyama, E.; Kamimura, M. Universality in Efimov-associated tetramers in ${ }^{4}$ He. Phys. Rev. A 2014, 90, 052514-10.

(28) Bressanini, D.; Zavaglia, M.; Mella, M.; Morosi, G. Quantum Monte Carlo investigation of small ${ }^{4} \mathrm{He}$ clusters with a ${ }^{3} \mathrm{He}$ impurity. J. Chem. Phys. 2000, 112, 717-722.

(29) Stipanović, P.; Vranješ Markić, L.; Boronat, J.; Kežić, B. Ground state of small mixed helium and spin-polarized tritium clusters: A quantum Monte Carlo study. J. Chem. Phys. 2011, 134, 054509-8.

(30) Yamashita, M. T.; Fedorov, D. V.; Jensen, A. S. Universality of Brunnian (N-body Borromean) four- and five-body systems. Phys. Rev. A 2010, 81, 063607-5.

(31) Boronat J.; Casulleras, J. Monte Carlo analysis of an interatomic potential for He. Phys. Rev. B 1994, 49, 8920-8930.

(32) Casulleras J.; Boronat, J. Unbiased estimators in quantum Monte Carlo methods: Application to liquid ${ }^{4}$ He. Phys. Rev. B 1995, 52, 3654-3661.

(33) Jeziorska, M.; Cencek, W.; Patkowski, B.; Jeziorski, B.; Szalewicz, K. Pair potential for helium from symmetry-adapted perturbation theory calculations and from supermolecular data. J. Chem. Phys. 2007, 127, 124303:1-13 .

(34) Casimir, H. B. G.; Polder, D. The Influence of Retardation on the London-van der Waals Forces. Phys. Rev. 1948, 73, 360-372.

(35) H. Suno, B. D. Esry, Phys. Rev. A 78, 062701 (2008).

(36) Aziz, R. A.; Slaman, M. J. An examination of ab initio results for the helium potential energy curve. J. Chem. Phys. 1991, 94, 8047-8053. 
(37) Kornilov, O. The quantum halo state of the helium trimer. Science 2015, 348, 498-499.

(38) Hiyama, E.; Kamimura, M. Variational calculation of ${ }^{4}$ He tetramer ground and excited states using a realistic pair potential. Phys. Rev. A 2012, 85, 022502-15.

(39) Bešlić, I.; Vranješ Markić, L.; Kilić, S. Diffusion Monte Carlo Study of the Small Mixed ${ }^{4} \mathrm{He}-{ }^{3} \mathrm{He}$ Clusters. J. Low Temp. Phys. 2006, 143, 257-265.

(40) Hiyama, E.; Kamimura, M. Four-Body Structure of $\Lambda$ Hypernuclei and ${ }^{4}$ He Tetramer System. Few-Body Syst. 2013, 54, 737-744.

(41) Ulrich, B.; Vredenborg, A.; Malakzadeh, A.; Schmidt, L. Ph. H.; Havermeier, T.; Meckel, M.; Cole, K.; Smolarski, M.; Chang, Z.; Jahnke, T. et al. Imaging of the Structure of the Argon and Neon Dimer, Trimer, and Tetramer. J. Phys. Chem. A 2011, 115, 6936-6941.

(42) Zinner, N. T.; Jensen, A. S. Comparing and contrasting nuclei and cold atomic gases. J. Phys. G: Nucl. Part. Phys. 2013, 40, 053101-63 


\section{Graphical TOC Entry}

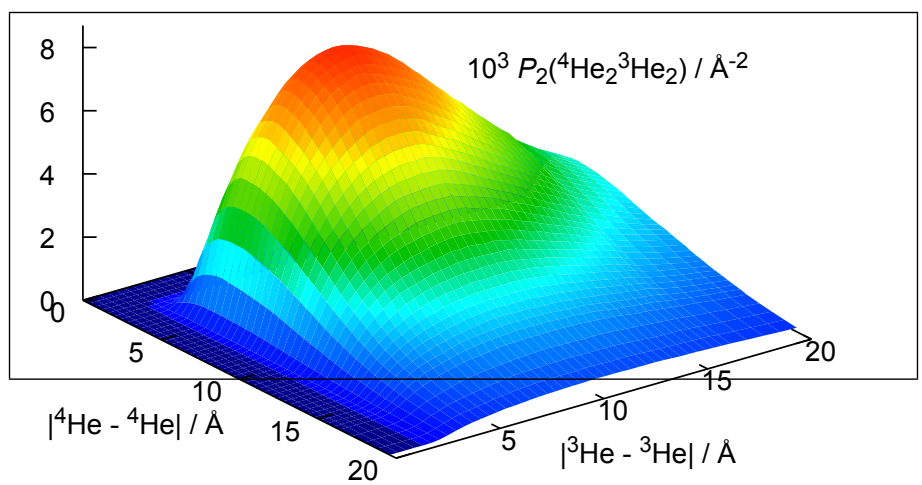

